\title{
10 Years of Acute Care Surgery: Experiences in a Single Tertiary University Hospital in Korea
}

\author{
Tae Hyun Kim a , Jung Yun Park b, Yun Tae Jung a, Seung Hwan Lee a, Myung Jae Jung c , Jae Gil Lee a \\ a Department of Surgery, Yonsei University College of Medicine, Seoul, Korea \\ ${ }^{b}$ Department of Trauma Surgery, Andong Medical Group hospital, Andong, Korea \\ ${ }^{c}$ Department of Surgery, Myongji Hospital, Goyang, Korea
}

\section{Article history:}

Received: November 5, 2019

Revised: November 13, 2019

Accepted: November 18, 2019

\section{${ }^{*}$ Corresponding Author:}

Jae Gil Lee

Department of Surgery, Yonsei University

College of Medicine, Seoul, Korea

03722, 50-1 Yonsei-ro, Seodaemun-gu,

Seoul, Korea

E-mail: jakii@yuhs.ac

\section{ORCID}

Tae Hyun Kim

https://orcid.org/0000-0001-7499-3269

Jung Yun Park

https://orcid.org/0000-0002-0129-9628

Yun Tae Jung

https://orcid.org/0000-0002-3583-7105

Seung Hwan Lee

https://orcid.org/0000-0001-7325-8262

Myung Jae Jung

https://orcid.org/0000-0002-9128-1180

Jae Gil Lee

https://orcid.org/0000-0002-1148-8035

\begin{abstract}
Purpose: Acute care surgery (ACS) has been shown to improve patient outcome and treatment efficiency in the U.S. ACS was introduced to the Department of Surgery, Yonsei University College of Medicine, Seoul to solve the problems associated with delays in surgical evaluation of non-trauma patients who needed emergency surgery, and to offer exposure to a wide variety of surgical cases to general surgical fellows and residents. The objective of this study was to describe the 10-year experience of the ACS department in a single center.
\end{abstract}

Methods: A retrospective chart review was conducted at the Department of Surgery, Yonsei University College of Medicine, Seoul, for all patients admitted from March 2008 to February 2018. Patients were grouped into either the trauma or non-trauma group, and were further classified according to their diagnosis, and the type of operations they had undergone.

Results: There was a total of 2,805 patients, including 1,001 trauma patients and 1,804 non-trauma patients. The average hospital length of stay was 14 days and the total in-hospital mortality rate was $3.6 \%$. Trauma mechanisms included blunt (92.6\%), penetrating $(7.0 \%)$ and burn $(0.4 \%)$ trauma. The majority of non-trauma patients were admitted for appendicitis (37.1\%), followed by cholecystitis (21.7\%). There was a total of 1,561 operations conducted. The most frequent operations were appendectomy (38.3\%) and cholecystectomy (19.5\%), followed by adhesiolysis (7.8\%).

Conclusion: A working ACS department has been implemented in a Korean medical center.

Keywords: critical care, hospital stay, surgery, trauma.

\section{Introduction}

The novel concept of acute care surgery (ACS) was initially proposed by the American Association for the Surgery of Trauma in 2005. The emerging specialty was composed of trauma, surgical critical care, and emergency general surgery [1]. Several factors have been proposed as contributing to the emergence of ACS in the U.S.; a national shortage of general surgeons [2], decreased interest in trauma or surgical critical care as a career option [3], and a medical blind spot of nontrauma patients who required emergency surgical treatment. ACS has been successfully adopted in many U.S. medical centers and has improved patient outcomes and treatment efficiency [4-8].

Patients who needed emergency surgical care traditionally had to wait for the general surgeon on-call, who was usually working in outpatient departments or performing elective surgery; In this traditional model, a blind spot was evident for surgical treatment of non-trauma patients before ACS was adopted [5]. This lack of prompt availability of the general surgeon for patients who needed emergency general surgery, delayed surgical evaluation, and treatment for these critically ill patients.

Trauma and surgical critical care as a specialty is not 
an attractive proposal for general surgery residents. The reasons for this may include the perception that trauma and surgical critical care is a non-operative field, has an increased medicolegal risk $[3,9,10]$, and has insufficient exposure to a wide variety of surgical cases. ACS includes not only trauma and surgical critical care, but also non-trauma emergency surgery and elective surgery. Therefore, ACS offers a better scope of practice to general surgery residents.

In 2008, the Department of Surgery, Yonsei University College of Medicine, Seoul, implemented the ACS model to address issues associated with delays of surgical evaluation of non-trauma patients who need emergency surgery, and to offer exposure to surgical residents, to a wide variety of surgical cases. This study aimed to describe the outcomes of practicing ACS for 10 years in a single tertiary hospital in Korea.

\section{Materials and Methods}

In March 2008 ACS was adopted by the Department of Surgery, Yonsei University College of Medicine, Seoul, and trauma and non-traumatic emergencies were covered by the ACS surgeon (Table 1). In the initial period (March 2008 to May 2014) of practice of ACS, 1 general surgeon (staff) and 1 surgical resident were dedicated to the ACS team. In March 2015, the newly formed Training Center for Trauma in the Department of Surgery had 3 dedicated surgeons appointed to trauma surgery, and the fellows of the trauma team were responsible for nontrauma emergencies. After February 2018, besides staffs, 2 surgical residents worked in the ACS team for 12 hours per day on rotation [dayshift ( $6 \mathrm{am}$ to $6 \mathrm{pm}$ ), nightshift ( $6 \mathrm{pm}$ to $6 \mathrm{am}$ )], and were responsible for both patients who had traumatic, and nontraumatic injuries. Table 1 provides a summary of weekly staffing schedules for the ACS model. The on-call system on weekdays is based on a 12-hour shift. During the dayshift, the ACS surgeon and 1 backup surgeon is on-call for non-trauma patients who need emergency surgery. For the nightshift, 1 scheduled surgeon [including the ACS surgeon (once or twice a week)] is on-call for non-trauma patients. Weekend shifts were based on a 24-hour shift where there was 1 scheduled on-call surgeon (except ACS surgeons). For trauma patients, 1 ACS surgeon and 1 backup ACS surgeon were on-call based on a 24-hour shift. Importantly, non-trauma patients who were admitted during the night ( $6 \mathrm{pm}$ to $6 \mathrm{am}$ ) and weekends, were excluded from the ACS data since they were admitted to the other Departments of General Surgery (not the Department of ACS). A retrospective analysis in a single center, university affiliated hospital from March 2008 to February 2018 was performed to review patients who were admitted from the Emergency Department or transferred to the ACS Department by other departments.

The data for analysis included demographic characteristics, diagnosis, and type of surgery. Patients were assigned to either trauma or non-trauma group, and were subclassified according to their diagnosis, and the operations they underwent. The trauma group was subcategorized into the type of trauma experienced; blunt, penetrating, and burn. The non-trauma group was subcategorized according to the diagnosis; appendicitis, cholecystitis, intestinal obstruction, gastroduodenal perforation, small bowel perforation, large bowel perforation, inflammatory bowel disease, gastrointestinal bleeding, intra-abdominal abscess, hernia, and

Table 1. Staffing schedule for acute care surgery models.

a) Non-trauma

\begin{tabular}{lcc}
\hline & Weekday & Weekend \\
\hline Day $(6: 00 \mathrm{am}-6: 00 \mathrm{pm})$ & 1 ACS surgeon & $\begin{array}{c}\text { 1 scheduled on call surgeon } \\
\text { (except ACS surgeon) }\end{array}$ \\
Night $(6: 00 \mathrm{pm}-6: 00 \mathrm{am})$ & $\begin{array}{c}\text { 1 scheduled on call surgeon } \\
\text { (including ACS surgeon) }\end{array}$ \\
\hline
\end{tabular}

b) Trauma

\begin{tabular}{lcc}
\hline & Weekday & Weekend \\
\hline Day $(6: 00 \mathrm{am}-6: 00 \mathrm{pm})$ & 1 ACS surgeon & 1 ACS surgeon \\
Night $(6: 00 \mathrm{pm}-6: 00 \mathrm{am})$ & 1 backup ACS surgeon & 1 backup ACS surgeon \\
\hline
\end{tabular}

c) Number of ACS surgeon

March 2008 - May 2014

June 2014 - Feb 2015

March 2015 - Feb 2018
1 ACS surgeon

2 ACS surgeon

3 trauma surgeons \& Fellows

ACS = acute care surgery. 
"others."

Patients who underwent surgery were grouped according to the type of operation they received; appendectomy, cholecystectomy, primary repair of hollow viscus, gastrectomy, small bowel resection, large bowel resection, diversion, hepatobilio-pancreatic surgery, resection of solid organ, adhesiolysis, bleeding control, hernia repair, and "others."

This study was approved by the Institutional Review Board of Yonsei University College of Medicine, Seoul (IRB no.: 4-2019-0927), and informed consent was waived due to the retrospective nature of the review of the medical records.

\section{Results}

During the 10-year study period, 2,805 total cases were analyzed and 1,001 were categorized as trauma, and 1,804 were categorized as non-trauma (Table 2). The mean age of the trauma group was 49.5 years, and the mean age of the nontrauma group was 53.8 years. The percentage of male patients in the trauma group was $66.9 \%$, and in the non-trauma group was $55.1 \%$. Hospital length of stay for the trauma group, and the non-trauma group were 22.38 days, and 9.42 days, respectively. The percentages of in-hospital mortality for the trauma group, and the non-trauma group were $5.6 \%$, and $2.5 \%$, respectively.

The trauma group were subclassified according to the type of traumatic injuries. Patients with blunt injuries comprised of $92.6 \%$ of all trauma patients, and patients with penetrating injuries, and burn injuries accounted for $7.0 \%$, and $0.4 \%$ respectively (Table 3 ). The non-trauma group was subclassified into 11 groups according to diagnosis. Appendicitis and cholecystitis accounted for $58.8 \%$ of all non-trauma patients, $37.1 \%$, and $21.7 \%$, respectively. Intestinal obstruction accounted $17.8 \%$ of all non-trauma patients.

Table 2. Characteristics of admitted patients for 10 years.

\begin{tabular}{llll}
\hline & Total $(n=2,805)$ & Trauma $(n=1,001)$ & Non-trauma $(n=1,804)$ \\
\hline Age $(\mathrm{y} ; \pm$ SD) & $52.3(19.52)$ & $49.5(19.28)$ & $53.8(19.49)$ \\
Male $:$ Female & $1,664: 1,141$ & $670: 331$ & $994: 810$ \\
H-LOS $(\mathrm{d} ; \pm$ SD) & $14.04(30.74)$ & $22.38(47.08)$ & $9.42(13.41)$ \\
In-hospital mortality & $102(3.6)$ & $56(5.6)$ & $46(2.5)$ \\
\hline
\end{tabular}

H-LOS = hospital length of stay.

Table 3. Numbers of admitted patients for 10 years.

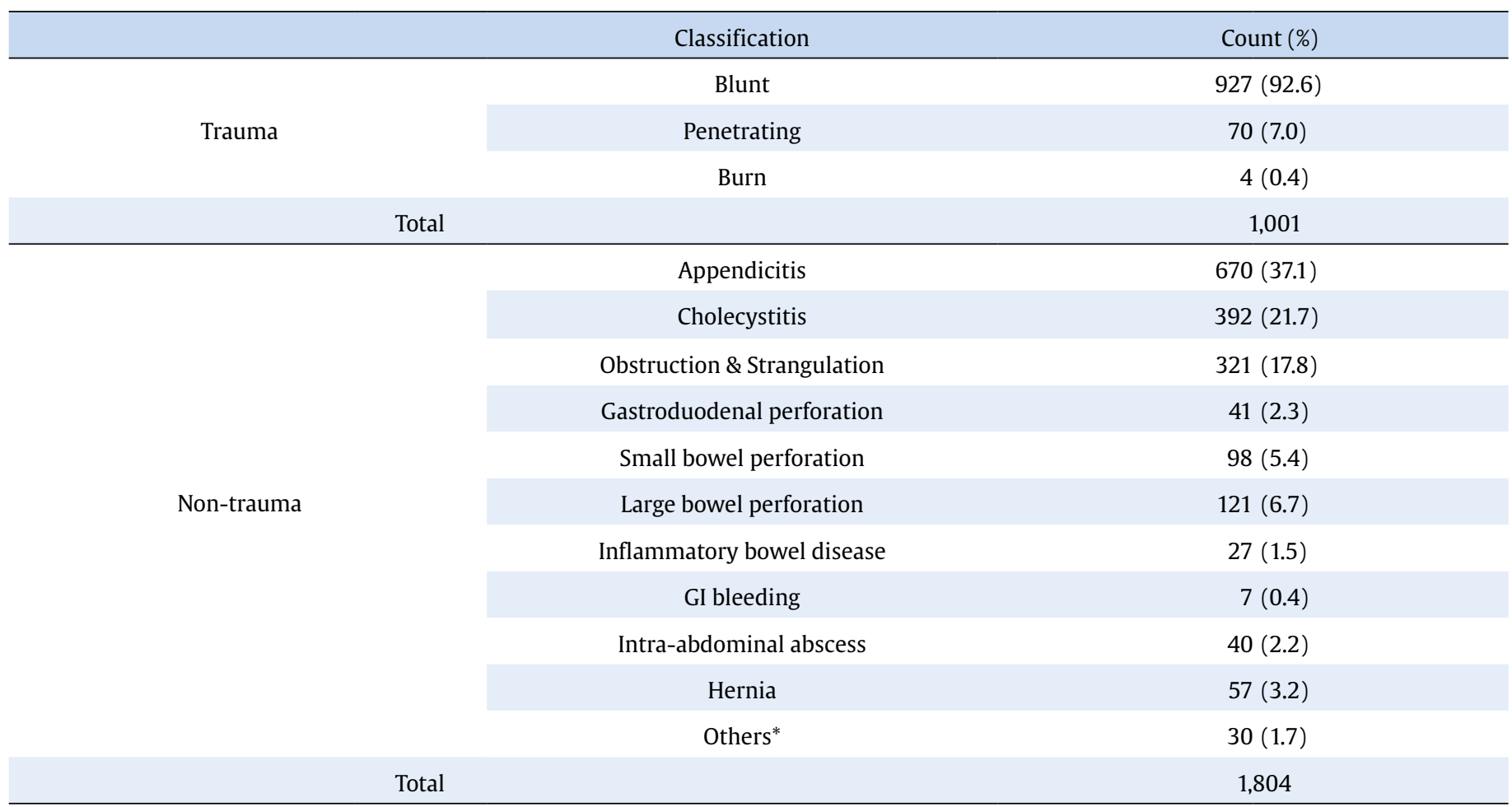

${ }^{*}$ Mesenteric venous thrombosis, pneumatosis intestinalis, bowel ischemia (without evidence of perforation) etc. 
Table 4. Numbers of operations for 10 years.

\begin{tabular}{|cc|}
\hline & Count (\%) \\
\hline Appendectomy & $598(38.3)$ \\
\hline Cholecystectomy & $304(19.5)$ \\
\hline Adhesiolysis \& bandlysis & $122(7.8)$ \\
\hline Bleeding control & $105(6.7)$ \\
\hline Small bowel resection & $97(6.2)$ \\
\hline Primary repair of hollow viscus & $88(5.6)$ \\
\hline Large bowel resection & $68(4.4)$ \\
\hline Hernia repair & $46(2.9)$ \\
\hline Diversion & $39(2.5)$ \\
\hline Resection of solid organ & $15(1.0)$ \\
\hline High blood pressure & $9(0.6)$ \\
\hline Gastrectomy & $8(0.5)$ \\
\hline Others* & $62(4.0)$ \\
\hline Total & 1,561 \\
\hline
\end{tabular}

* Intraabdominal drainage, abdominal wall closure, hematoma evacuation, foreign body removal, etc.

(A)

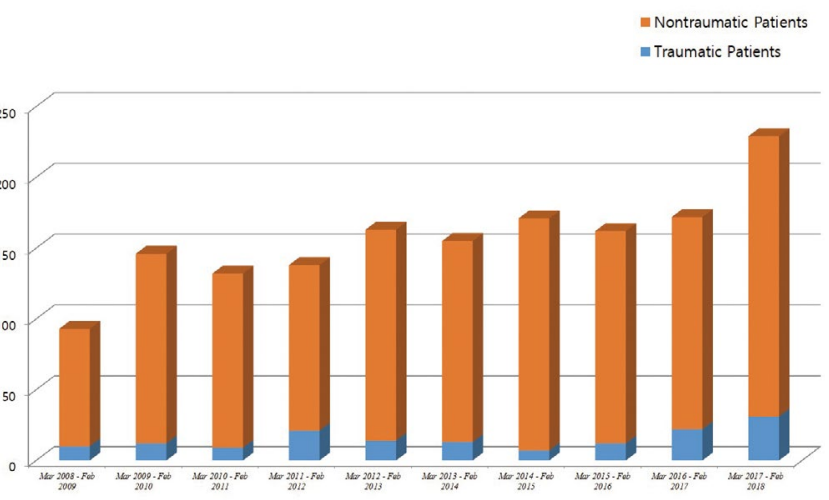

(B)

- Appendectomy - Cholecystectomy

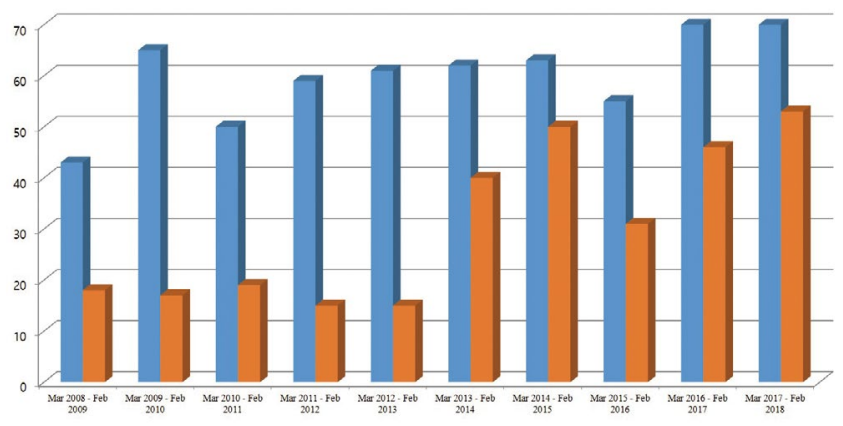

Figure 1. Annual number of surgical procedures. (A) Comparing nontraumatic cases with traumatic cases, and (B) comparing the number of appendectomy cases with the number of cholecystectomy cases.
There were $55.6 \%$ of patients who were admitted to the Department of ACS who underwent surgical treatment (Table 4). There were 598 out of 1,561 cases $(38.3 \%)$ that were appendectomies. The appendectomy was typically performed by laparoscopic approach (96.2\%). The second most common cases were cholecystectomies, accounting for $19.5 \%$ of cases. This was followed by adhesiolysis and bandlysis (7.8\%) surgery for intestinal obstruction. Most cases of bleeding control (6.7\% of all surgical cases) were caused by blunt traumatic event.

Figure 1 shows the number of annual surgical procedures conducted by the ACS team. The number of surgical procedures for trauma patients are relatively constant. On the other hand, the data showed an upward trend in the annual number of non-trauma emergency surgeries. The numbers of appendectomy cases were generally constant in the 10 -year period.

\section{Discussion}

In the Department of Surgery, Yonsei University College of Medicine, Seoul, before implementing the ACS model, a general surgeon who was on-call over a 24 -hour period, was in charge of non-trauma patients who needed emergency surgical treatment. These general surgeons who were on-call were typically preoccupied by other activities such as elective surgery, and working in Out-Patient Departments therefore delays in surgical evaluation and treatment occurred. These concerns prompted the adoption of the new concept of ACS in 2008 as a new specialty. It was composed of trauma, surgical critical care, and emergency surgery. Since the implementation of the ACS model in 2008, an ACS team included an attending surgeon in charge of non-trauma patients and another attending surgeon in charge of trauma patients during the daytime and on weekdays. This provided timely surgical evaluation and management for critically ill patients. In early period from March 2008 to May 2014, only 1 surgeon was dedicated as ACS surgeon. From 2014, the institution was appointed as The Training Center for Trauma Surgeons, so 3 surgeons performed ACS surgery. However, due to the limitation of only having a single surgeon and a training center, comprehensive emergency surgery was not covered by the ACS team.

The ACS team was composed of not only the ACS surgeons, but also fellows and 2 general surgical residents, in training. Fellows worked 24-hour shifts, and the 2 residents worked 12 hours per day on rotation. The number of ACS surgeons and residents involved in the ACS team was restricted by the systems and policies in place at the hospital. Therefore, optimization of the ACS model is restricted by working to hospital and governmental policies. This is a nationwide issue 
whereby the government inadvertently affects the national medical system under the guise of managing the national health insurance program.

The durability of ACS as a specialty in Korean hospitals depends on the numbers of general surgical residents it attracts. ACS includes trauma, surgical critical care, emergency surgery and elective surgery [11]. In 2012, a survey was conducted to examine the opinions of general surgical residents in the U.S. regarding training and careers in ACS. There were $46 \%$ of respondents who considered pursuing an ACS fellowship which was in stark contrast to previous surveys which reported only $6 \%$ to $18 \%$ of surgical residents would consider a fellowship in trauma surgery [12]. Typically, this lack of interest in pursuing trauma and surgical critical care has been due to the perception that this area is a non-operative field. However, by including non-traumatic injury emergency surgery and elective surgery, ACS offers a better scope of surgical practice in terms of numbers and variety of surgical cases that may be attractive to general surgical residents.

The data from this study showed that acute appendicitis and cholecystitis were the most common non-traumatic conditions requiring surgical emergency accounting for $37.1 \%$ and $21.7 \%$ of all the non-trauma patients, respectively. In the U.S., research data on the medical outcomes of ACS and its effectiveness has been accumulating since the implementation of ACS in 2005. Cubas et al [5] compared the ACS model with the traditional home-call model and showed that the ACS model had better medical outcomes and cost effectiveness for patients who were diagnosed with acute appendicitis or cholecystitis. Lau et al [7] reported that ACS improved timeliness of medical care and reduced hospital stay for patients with acute cholecystitis. The ACS care service model led to a better outcome overall for patients who were diagnosed with acute appendicitis or cholecystitis, the most common surgical emergencies observed in U.S. Emergency Departments in the scope of patient benefits and health care cost benefits.

Most general surgeons in Korea recognize that a specialty for emergency surgery and surgical critical care is necessary [13]. The worry is that the traditional general surgery model lacks sufficient resources and expertise for emergency surgery and surgical critical care. ACS in Korea is emerging, although there are government and social issues to be solved before the ACS model can be used nationwide in Korea. However, the data reported from a single-regional trauma center study suggested a working setup of an ACS system in Korea could be achieved [14].

This study has several limitations. Firstly, the results describe a single center with a partially-adapted ACS system. Therefore, the results could not be generalized to other hospitals in
Korea. Secondly, the experience and the number of emergency surgeries may vary considerably according to resources such as manpower, availability of operating room, and backup anesthesiologists. However, the data from this review of 10-years of experience of using the ACS model in a Korean medical center, is valuable. It is the first long term massive data set of the ACS model in Korea. By providing a timely diagnosis and surgical treatment, the ACS system is a working model implemented in this institution. Analysis of this data set in the future allows a comparison of patient outcome, treatment efficiency, and cost effectiveness of ACS with the traditional general surgery model previously used at this institution.

\section{Conflicts of Interest}

The authors have no conflicts of interest to declare.

\section{References}

[1] Committee to Develop the Reorganized Specialty of Trauma, Surgical Critical Care, and Emergency Surgery Emergency, Acute care surgery. Trauma, critical care, and emergency surgery. J Trauma 2005;58(3):614-6.

[2] Cohn SM, Price MA, Villarreal CL. Trauma and surgical critical care workforce in the United States: A severe surgeon shortage appears imminent. J Am Coll Surg 2009;209(4):446-52.e4.

[3] Hadzikadic L, Burke PA, Esposito TJ, Agarwal S. Surgical resident perceptions of trauma surgery as a specialty. Arch Surg 2010;145(5):44550.

[4] Britt RC, Weireter LJ, Britt LD. Initial implementation of an acute care surgery model: Implications for timeliness of care. J Am Coll Surg 2009;209(4):421-4.

[5] Cubas RF, Gomez NR, Rodriguez S, Wanis M, Sivanandam A, Garberoglio CA. Outcomes in the management of appendicitis and cholecystitis in the setting of a new acute care surgery service model: Impact on timing and cost. J Am Coll Surg 2012;215(5):715-21.

[6] Earley AS, Pryor JP, Kim PK, Hedrick JH, Kurichi JE, Minogue AC, et al. An acute care surgery model improves outcomes in patients with appendicitis. Ann Surg 2006;244(4):498-504.

[7] Lau B, Difronzo LA. An acute care surgery model improves timeliness of care and reduces hospital stay for patients with acute cholecystitis. Am Surg 2011;77(10):1318-21.

[8] Lehane CW, Jootun RN, Bennett M, Wong S, Truskett P. Does an acute care surgical model improve the management and outcome of acute cholecystitis? ANZ J Surg 2010;80(6):438-42.

[9] Richardson JD, Miller FB. Will future surgeons be interested in trauma care? Results of a resident survey. J Trauma 1992;32(2):229-33; discussion 233-5.

[10] Esposito TJ, Maier RV, Rivara FP, Carrico CJ. Why surgeons prefer not to care for trauma patients. Arch Surg 1991;126(3):292-7.

[11] Peitzman AB, Sperry JL, Kutcher ME, Zuckerbraun BS, Forsythe RM, Billiar TR, et al. Redefining acute care surgery: Surgical rescue. J Trauma Acute Care Surg 2015;79(2):327.

[12] Coleman JJ, Esposito TJ, Rozycki GS, Feliciano DV. Acute care surgery: Now that we have built it, will they come? J Trauma Acute Care Surg 2013;74(2):463-8; discussion 468-9.

[13] Park CI, Kim JH, Park SJ, Kim SH, Kim HH, Hong SK, et al. Acute Care Surgery: Implementation in Korea. J Acute Care Surg 2018;8(2):51-8.

[14] Jang JY, Shim H, Kim JH, Hong SK, Bae KS. Current status of the Korean acute care surgery system for non-traumatic abdominal emergency surgery: A single-regional trauma center study. J Korean Med Assoc 2019;62(2):130-4 